\title{
The Effect of Aging and Small-Vessel Disease Burden on Hematoma Location in Patients with Acute Intracerebral Hemorrhage
}

\author{
Yuki Sakamoto Takahiro Sato Chikako Nito Yasuhiro Nishiyama \\ Satoshi Suda Noriko Matsumoto Junya Aoki Tomonari Saito \\ Kentaro Suzuki Takehiro Katano Kazumi Kimura \\ Department of Neurology, Graduate School of Medicine, Nippon Medical School, Tokyo, Japan
}

\section{Keywords \\ Intracerebral hemorrhage · Aging · Etiology · Magnetic resonance imaging $\cdot$ Small-vessel disease}

\begin{abstract}
Introduction: Intracerebral hemorrhage $(\mathrm{ICH})$ is a devastating hemorrhagic event and is associated with high mortality or severe neurological sequelae. Age-associated differences in hematoma location for nonlobar ICH are not well known. The aims of the present study were to elucidate the relationship between age and hematoma location and to assess the differences in small-vessel disease (SVD) burden as a potential surrogate marker for longstanding hypertension among various hematoma locations. Methods: From September 2014 through July 2019, consecutive patients with acute, spontaneous $\mathrm{ICH}$ were retrospectively enrolled from a prospective registry. Magnetic resonance imaging was performed during admission, and the total SVD burden score (including microbleeds, lacunes, enlarged perivascular spaces, and white matter hyperintensities) was calculated. The relationships of hematoma location with aging and SVD burden were assessed by using multivariate logistic regression
\end{abstract}

analyses. Results: A total of 444 patients (156 women [35\%]; median age 69 [interquartile range 59-79] years; National Institutes of Health Stroke Scale score 9 [17][3-17]) were enrolled in the present study. Multivariate logistic regression analyses showed that advanced age was independently associated with thalamic (odds ratio [OR]: 1.48, 95\% confidence interval $[\mathrm{Cl}]: 1.19-1.84, p<0.001$ for 10 -year increment) and lobar hemorrhage (OR: 1.58, 95\% Cl: 1.19-2.09, $p=0.002$ ) and was independently and negatively related to putaminal hemorrhage (OR: $0.55,95 \% \mathrm{Cl}: 0.44-0.68, p<$ $0.001)$. The total SVD burden score was independently and positively associated with thalamic hemorrhage (OR: 1.27, $95 \% \mathrm{Cl}: 1.01-1.59, p=0.045)$ and negatively with lobar hemorrhage (OR: $0.74,95 \% \mathrm{Cl}: 0.55-0.99, p=0.042)$, even after adjusting by age, but not with putaminal hemorrhage (OR: 0.91, 95\% Cl: 0.73-1.14, $p=0.395)$. Conclusion: Putaminal, thalamic, and lobar hemorrhages are prone to occur in specific ages and SVD states: putaminal in young patients, thalamic in old and high SVD burden patients, and lobar hemorrhages in old and low SVD burden patients. Susceptibility to bleeding with aging or severe SVD accumulation seems to differ considerably among brain locations.

(C) 2021 S. Karger AG, Base

\footnotetext{
Karger ${ }^{\prime}=$
} 


\section{Introduction}

Intracerebral hemorrhage (ICH) is a devastating hemorrhagic event that is associated with high mortality and severe neurological sequelae [1]. The incidence of ICH rises with age, and although the annual incidence of $\mathrm{ICH}$ has decreased through the past few decades, the absolute number of patients has increased, especially elderly individuals in developed countries [2]. The mean age of patients with first-ever ICH is rising [2], and the number of old $\mathrm{ICH}$ patients is predicted to continue to rise [3]. Elderly ICH patients had more baseline comorbidities and antithrombotic treatments, and mortality was higher than for young ICH patients [3-7]. To establish appropriate management strategies for elderly ICH patients is one of the future challenges [8].

Understanding the clinical characteristics and mechanisms of elderly ICH patients is one of the bases for developing approaches for such patients. Clinical features of ICH, in terms of hematoma volume, hematoma location, and blood pressure (BP) at onset, are considered to differ between young and old ICH patients $[9,10]$. Lobar hemorrhage is reported to increase in elderly patients, mainly in association with cerebral amyloid angiopathy (CAA) [11]. However, age-associated differences in hematoma location for nonlobar ICH are not well known. A few past studies suggested that thalamic or cerebellar hemorrhage was more frequently observed than putaminal hemorrhage in aged ICH patients $[6,7,10,12]$, but almost all of the results were from univariate analyses. Elucidating the relationship between aging and hematoma location can be a clue for developing age-specific ICH prevention and treatment strategy; indeed, some hemorrhage locations including thalamus were reported to be associated with worse outcomes [13]. We hypothesized that thalamic hemorrhage is more frequent as age advances because thalamic hemorrhage occurs based on longstanding hypertension, whereas putaminal hemorrhage is based on an abrupt BP increment [14]. Cerebral small-vessel disease (SVD) due to microangiopathies not related to CAA is known to be caused by longstanding hypertension $[15,16]$, and it is reported to increase with age [17], but the association between SVD and hematoma location has not been well studied. The aims of the present study were to elucidate the relationship between age and hematoma location and to assess the differences in SVD burden as a potential surrogate marker for longstanding hypertension in various hematoma locations.

\section{Methods}

\section{Subjects}

From September 2014 through July 2019, consecutive patients with acute ( $<7$ days from onset), spontaneous ICH admitted to our stroke unit (SU) were retrospectively enrolled from a prospective registry [18]. Patients with secondary ICH and pure intraventricular hemorrhage were excluded. This study was approved by the institutional Ethics Committee (No. 26-10-398). Written, informed consent was obtained from all patients or their next of kin.

\section{Clinical Characteristics}

Clinical background characteristics, including sex, age, cardiovascular risk factors, and past medical history, were recorded on admission. Cardiovascular risk factors were defined as follows: (1) hypertension, history of using antihypertensive agents, systolic $\mathrm{BP} \geq 140 \mathrm{~mm} \mathrm{Hg}$, or diastolic BP $\geq 90 \mathrm{~mm} \mathrm{Hg}$ before or $\geq 2$ weeks after stroke onset; (2) diabetes mellitus, use of hypoglycemic agents, random glucose level $\geq 200 \mathrm{mg} / \mathrm{dL}$, or glycosylated hemoglobin $\geq 6.5 \%$ on admission; (3) hyperlipidemia, use of antihyperlipidemic agents, serum total cholesterol level $\geq 220 \mathrm{mg} /$ $\mathrm{dL}$, or low-density lipoprotein cholesterol level $\geq 140 \mathrm{mg} / \mathrm{dL}$; (4) current smoker; and (5) daily alcohol intake. Neurological severity was assessed by using the National Institutes of Health Stroke Scale (NIHSS), and functional outcomes were evaluated by using the modified Rankin scale (mRS). Anthropometric measurements and routine blood biochemistry examinations were performed on admission. Hematoma location was classified by 2 experienced stroke neurologists (Y.S. and T.S.) by consensus as putamen, thalamus, lobar, caudate, brainstem, and cerebellum. When a hematoma occupied $>1$ location, the hematoma location was determined to be the one that contained the largest portion of the hematoma. Hematoma volume was evaluated with noncontrast computed tomography on admission by using the $\mathrm{ABC} / 2$ method.

\section{Calculating Total SVD Burden Score}

Multimodal 1.5-T magnetic resonance imaging (MRI, Echelon Oval; Hitachi Medical Systems, Tokyo, Japan) including fluid-attenuated inversion recovery (TR/TE, 10,000/120 ms; TI, 2,500 ms; field of view, $24 \mathrm{~cm}$; acquisition matrix, $288 \times 224$; and slice thickness, $4.5 \mathrm{~mm}$, with a $2.5-\mathrm{mm}$ intersection gap), T2-weighted imaging (TR/TE 4,200/117.52 ms), and T2*-weighted imaging (TR/ TE, 480/18 ms; field of view, $24 \mathrm{~cm}$; acquisition matrix, $256 \times 204$; and slice thickness, $4.5 \mathrm{~mm}$, with a $2.5-\mathrm{mm}$ intersection gap) was performed during admission. The imaging evidence of SVD was assessed based on the presence and severity of white matter hyperintensities (WMHs), cerebral microbleeds, lacunes, and enlarged perivascular spaces. These changes were assessed based on the Standards for Reporting Vascular Changes in Neuroimaging (STRIVE) criteria [19]. The severity of WMHs was assessed by using the Fazekas scale [20], the severity of enlarged perivascular spaces was scored by using a semiquantitative score [21], and these scores were evaluated on the side contralateral to the hematoma. To assess the association between SVD and hematoma location, the total SVD burden score [22] (0-4) was calculated by adding 1 point for each SVD feature: severe periventricular (Fazekas grade 2 or 3 ) and/or deep WMHs (Fazekas grade 3); presence of microbleed; presence of lacune; and prominent enlarged perivas- 
Table 1. Baseline clinical characteristics by age-group

\begin{tabular}{|c|c|c|c|c|c|c|}
\hline Variable & $\begin{array}{l}\text { Total } \\
(n=444)\end{array}$ & $\begin{array}{l}\text { Young } \\
(<65 \text { yrs }) \\
(n=161)\end{array}$ & $\begin{array}{l}\text { Young-old } \\
(65-74 \text { yrs }) \\
(n=130)\end{array}$ & $\begin{array}{l}\text { Old-old } \\
(75-84 \text { yrs }) \\
(n=100)\end{array}$ & $\begin{array}{l}\text { Oldest-old } \\
(\geq 85 \text { yrs }) \\
(n=53)\end{array}$ & $p$ value \\
\hline Body mass index, median (IQR), $\mathrm{kg} / \mathrm{m}^{2}$ & $22.4(20.3-25.1)$ & $24.7(21.7-28.2)$ & $23.0(20.9-25.1)$ & $21.2(19.0-23.1)$ & $21.3(19.1-23.4)$ & $<0.001$ \\
\hline Prior history of ischemic stroke, $n(\%)$ & $46(10)$ & $14(9)$ & $11(9)$ & $14(14)$ & $7(13)$ & 0.411 \\
\hline Prior history of hemorrhagic stroke, $n$ (\%) & $36(8)$ & $16(10)$ & $7(5)$ & $12(12)$ & $1(2)$ & 0.078 \\
\hline Hypertension & $378(85)$ & $139(86)$ & $113(87)$ & $87(87)$ & $39(74)$ & 0.095 \\
\hline Diabetes mellitus & $81(18)$ & $27(17)$ & $28(22)$ & $17(17)$ & $9(17)$ & 0.719 \\
\hline Hyperlipidemia & $124(28)$ & $49(30)$ & $30(23)$ & $35(35)$ & $10(19)$ & 0.083 \\
\hline Current smoking & $139(31)$ & $74(46)$ & $41(32)$ & $22(22)$ & $2(4)$ & $<0.001$ \\
\hline Daily alcohol intake & $228(51)$ & $109(68)$ & $70(54)$ & $35(35)$ & $14(26)$ & $<0.001$ \\
\hline Premorbid antithrombotic treatment, $n(\%)$ & $105(24)$ & $18(11)$ & $36(28)$ & $34(34)$ & $17(32)$ & $<0.001$ \\
\hline Preadmission mRS score, median (IQR) & $0(0-0)$ & $0(0-0)$ & $0(0-0)$ & $0(0-1)$ & $1(0-3)$ & $<0.001$ \\
\hline Putamen & $158(36)$ & $87(54)$ & $40(31)$ & $19(19)$ & $12(23)$ & $<0.001$ \\
\hline Thalamus & $156(35)$ & $42(26)$ & $54(42)$ & $39(39)$ & $21(40)$ & 0.026 \\
\hline Lobar & $78(18)$ & $13(8)$ & $20(15)$ & $32(32)$ & $13(25)$ & $<0.001$ \\
\hline Caudate nucleus & $7(2)$ & $2(1)$ & $1(1)$ & $2(2)$ & $2(4)$ & 0.489 \\
\hline Brainstem & $24(5)$ & $14(9)$ & $5(4)$ & $3(3)$ & $2(4)$ & 0.143 \\
\hline Cerebellum & $21(5)$ & $3(2)$ & $10(8)$ & $5(5)$ & $3(6)$ & 0.134 \\
\hline Initial hematoma volume, median (IQR), mL & $8.2(3.2-21.8)$ & $9.5(3.6-21)$ & $7.2(2.7-15)$ & $7.0(3.2-22)$ & $7.4(3.0-14)$ & 0.235 \\
\hline \multicolumn{7}{|l|}{ Biochemistry sign at admission, median (IQR) } \\
\hline Hemoglobin, g/dL & $13.9(12.6-15.1)$ & $14.8(13.7-15.6)$ & $14.4(13.2-15.4)$ & $13.3(11.9-14.1)$ & $11.9(10.9-13.7)$ & $<0.001$ \\
\hline Platelet count, $10^{4} / \mu \mathrm{L}$ & $21.0(17.4-25.0)$ & $22.2(19.1-27.3)$ & $20.4(17.5-23.4)$ & $19.6(16.9-24.6)$ & $20.6(14.8-26.2)$ & 0.001 \\
\hline Total cholesterol, mg/dL* & $190(166-216)$ & $195(174-221)$ & $188(166-209)$ & $180(159-208)$ & $184(158-224)$ & 0.033 \\
\hline $\mathrm{eGFR}, \mathrm{mL} / \mathrm{min}$ & $69(57-83)$ & $74(60-88)$ & $69(59-84)$ & $62(51-73)$ & $58(40-78)$ & $<0.001$ \\
\hline mRS score at discharge, median (IQR) & $4(2-5)$ & $3(2-4)$ & $4(2-5)$ & $4(3-5)$ & $4(3-5)$ & 0.010 \\
\hline
\end{tabular}

mRS, modified Rankin scale; BP, blood pressure; NIHSS, National Institutes of Health Stroke Scale; eGFR, estimated glomerular filtration rate; IQR, interquartile range. $*$ Data missing in 20 patients.

cular space (grades 2-4) in the basal ganglia. Each component of the total SVD burden score was assessed by Y.S. and S.S. separately for calculating inter-rater reliability and then decided by consensus in cases of nonagreement.

\section{Statistical Analyses}

All patients were divided into 4 groups based on their age at ICH onset: young ( $<65$ years), young-old (65-74 years), old-old (75-84 years), and oldest-old ( $\geq 85$ years). First, clinical background characteristics were compared among the 4 groups by using univariate analyses. Second, the associations between hematoma locations and advanced age were analyzed by using multivariate logistic regression analyses with a backward stepwise selection procedure. Each hematoma location was entered as a dependent variable, and variables shown in Table 1 (except the NIHSS and $\mathrm{mRS}$ score at discharge because these variables were consequences of, rather than factors associated with, hematoma location) were included in the models as independent variables, and a backward selection procedure was performed using $p>0.1$ of the likelihood ratio test for exclusion. Next, to assess the effect of SVD burden on hematoma location, clinical characteristics including the total SVD burden score were compared among putaminal, tha- lamic, lobar, and other location (caudate, brainstem, and cerebellum) hemorrhage patients. Patients with caudate, brainstem, and cerebellar hemorrhage were analyzed together due to their small sample size. Finally, multivariate logistic regression analyses were performed to identify independent factors associated with various hematoma locations. All variables identified on univariate analyses with values of $p<0.1$ including total SVD burden score, but not the initial NIHSS score and diastolic BP, were entered into the models. The initial NIHSS score was excluded because this variable was a consequence of, rather than a factor associated with, hematoma location. Diastolic BP was excluded because it was highly correlated with systolic BP. The relationship between hematoma location and the total SVD burden score was also tested in first-ever stroke patients. Moreover, because ICH due to CAA was not completely ruled out in the present study and the association between CAA and microbleeds, especially strictly lobar microbleeds, was reported $[23,24]$, multivariate analyses using the modified SVD score which removed the effect of microbleeds or strictly lobar microbleeds (SVD score only for enlarged perivascular space, WMH, and lacune, or not add 1 point for microbleeds when microbleed was located only in lobar, respectively) were conducted, for sensitivity analysis. The relative risks for specific hematoma locations 
Table 2. Results of binary logistic regression models for factors associated with (a) putaminal hemorrhage, (b) thalamic hemorrhage, and (c) lobar hemorrhage

\begin{tabular}{llr}
\hline Variable & OR $(95 \% \mathrm{CI})$ & $p$ values \\
\hline a Putaminal hemorrhage & & \\
Age, per 10 years & $0.55(0.44-0.68)$ & $<0.001$ \\
Body mass index, per $1 \mathrm{~kg} / \mathrm{m}^{2}$ & $0.95(0.89-1.01)$ & 0.078 \\
Hypertension & $0.53(0.25-1.13)$ & 0.100 \\
Premorbid antithrombotic treatment & $0.59(0.33-1.08)$ & 0.088 \\
Preadmission mRS & $1.30(1.04-1.63)$ & 0.020 \\
Hematoma volume, per $10 \mathrm{~mL}$ & $1.11(1.02-1.20)$ & 0.011 \\
\hline b Thalamic hemorrhage & & \\
Age, per 10 years & $1.48(1.19-1.84)$ & $<0.001$ \\
Past history of vascular disease & $5.01(1.51-16.6)$ & 0.009 \\
Systolic BP on arrival, per $10 \mathrm{~mm} \mathrm{Hg}$ & $1.09(1.00-1.17)$ & 0.038 \\
Hematoma volume, per $10 \mathrm{~mL}$ & $0.57(0.46-0.70)$ & $<0.001$ \\
Hemoglobin, per $1 \mathrm{~g} / \mathrm{dL}$ & $1.13(0.99-1.29)$ & 0.058 \\
Platelet count, per $10^{4} / \mu \mathrm{L}$ & $1.05(1.01-1.09)$ & 0.014 \\
\hline c Lobar hemorrhage & & \\
Age, per 10 years & $1.58(1.19-2.09)$ & 0.002 \\
Female sex & $2.43(1.27-4.66)$ & 0.008 \\
Body mass index, per $1 \mathrm{~kg} / \mathrm{m}^{2}$ & $1.13(1.04-1.22)$ & 0.003 \\
Hypertension & $2.24(1.04-4.79)$ & 0.003 \\
Systolic BP on arrival, per $10 \mathrm{~mm} \mathrm{Hg}$ & $0.85(0.77-0.94)$ & 0.002 \\
Hematoma volume, per $10 \mathrm{~mL}$ & $1.36(1.22-1.51)$ & $<0.001$ \\
Total cholesterol, per $100 \mathrm{mg} / \mathrm{dL}$ & $0.51(0.24-1.08)$ & 0.078 \\
\hline
\end{tabular}

The variables identified by the backward selection procedure are listed. OR, odds ratio; $\mathrm{CI}$, confidence interval; mRS, modified Rankin scale; BP, blood pressure. are expressed as odds ratios (ORs) with $95 \%$ confidence intervals (CIs). Univariate analyses were performed by using the $\chi^{2}$ test, Fisher's exact test, or the Mann-Whitney $U$ test, as appropriate. The data are presented as median values (interquartile range [IQR]) or numbers (\%). All statistical analyses were performed by using SPSS version 23.0 software (IBM SPSS Inc., Armonk, NY, USA). Results were considered significant at $p<0.05$.

Data Availability

Data are available upon reasonable request.

\section{Results}

Overall, 464 consecutive patients with ICH were admitted to our SU during the study period. Of these, 16 patients diagnosed as having secondary ICH due to cerebral neoplasms, bleeding diathesis, arteriovenous malformations, moyamoya disease, infective endocarditis, minor trauma, or cerebral venous thrombosis were excluded. Four patients were also excluded due to pure intraventricular hemorrhage. Finally, 444 patients (156 women [35\%]; median age, 69 [IQR 59-79] years; NIHSS score, 9 [3-17]; median time from symptom onset to admission,
$2.60[1.01-12.0]$ hours) were enrolled in the present study (see online suppl. Fig. 1; for all online suppl. material, see www.karger.com/doi/10.1159/000515411). Of these 444 patients, 161 (36\%) were classified as the young group, 130 (29\%) were in the young-old group, 100 (23\%) were in the old-old group, and $53(12 \%)$ were in the oldest-old group. Table 1 shows the baseline clinical characteristics of the 4 groups. The proportions of female sex $(p<0.001)$, having a prior history of vascular disease $(p<0.001)$, current smoker $(p<0.001)$ and drinker $(p<0.001)$, and receiving premorbid antithrombotic treatment $(p<0.001)$, as well as the values of the body mass index $(p<0.001)$, preadmission mRS score $(p<0.001)$, time from onset to admission $(p=0.011)$, systolic $(p=0.003)$ and diastolic $(p<0.001)$ BPs, laboratory data, and mRS score at discharge $(p=$ 0.010 ), were significantly different among the 4 groups. The rate of putaminal hemorrhage tended to decrease, whereas thalamic and lobar hemorrhages tended to increase with advanced age ( $p=0.026$, Fig. 1 ). Multivariate logistic regression analyses showed that advanced age was independently and negatively associated with putaminal hemorrhage (OR: $0.55,95 \%$ CI: $0.44-0.68$ for 10 -year increment, $p<0.001$, Table $2 \mathrm{a}$ ), and positively with thalam- 
Table 3. Multivariate logistic regression model for associated factors with putaminal, thalamic, and lobar hemorrhages

\begin{tabular}{|c|c|c|c|c|c|c|}
\hline \multirow[t]{3}{*}{ Independent variable } & \multicolumn{6}{|c|}{ Dependent variable } \\
\hline & \multicolumn{2}{|c|}{ putaminal hemorrhage } & \multicolumn{2}{|c|}{ thalamic hemorrhage } & \multicolumn{2}{|l|}{ lobar hemorrhage } \\
\hline & OR (95\% CI) & $p$ value & OR $(95 \% \mathrm{CI})$ & $p$ value & OR (95\% CI) & $p$ value \\
\hline Female sex & $1.08(0.60-1.94)$ & 0.798 & $1.41(0.78-2.56)$ & 0.260 & $1.28(0.63-2.59)$ & 0.491 \\
\hline Age, per 10 years & $0.59(0.47-0.76)$ & $<0.001$ & $1.23(0.96-1.57)$ & 0.095 & $1.58(1.16-2.16)$ & 0.004 \\
\hline Hypertension & $0.49(0.23-1.04)$ & 0.064 & $1.29(0.63-2.63)$ & 0.489 & $1.87(0.85-4.07)$ & 0.118 \\
\hline Current smoking & $0.75(0.43-1.31)$ & 0.313 & $1.37(0.78-2.42)$ & 0.278 & $0.98(0.46-2.11)$ & 0.968 \\
\hline Daily alcohol intake & $1.76(1.02-3.04)$ & 0.044 & $1.13(0.64-1.98)$ & 0.681 & $0.58(0.29-1.15)$ & 0.117 \\
\hline Premorbid antithrombotic treatment & $0.53(0.27-1.05)$ & 0.067 & $1.11(0.61-2.02)$ & 0.742 & $1.34(0.64-2.80)$ & 0.435 \\
\hline Systolic BP on arrival, per $10 \mathrm{~mm} \mathrm{Hg}$ & $0.95(0.87-1.03)$ & 0.186 & $1.05(0.97-1.15)$ & 0.216 & $0.90(0.81-0.99)$ & 0.039 \\
\hline Hematoma volume, per $10 \mathrm{~mL}$ & $1.06(0.97-1.17)$ & 0.201 & $0.50(0.38-0.67)$ & $<0.001$ & $1.43(1.26-1.63)$ & $<0.001$ \\
\hline Hemoglobin, per $1 \mathrm{~g} / \mathrm{dL}$ & $1.00(0.86-1.15)$ & 0.966 & $1.05(0.91-1.22)$ & 0.495 & $0.99(0.83-1.18)$ & 0.915 \\
\hline Total SVD score, per 1 point & $0.91(0.73-1.14)$ & 0.395 & $1.27(1.01-1.59)$ & 0.045 & $0.74(0.55-0.99)$ & 0.042 \\
\hline
\end{tabular}

BP, blood pressure; SVD, small-vessel disease; OR, odds ratio; CI, confidence interval.

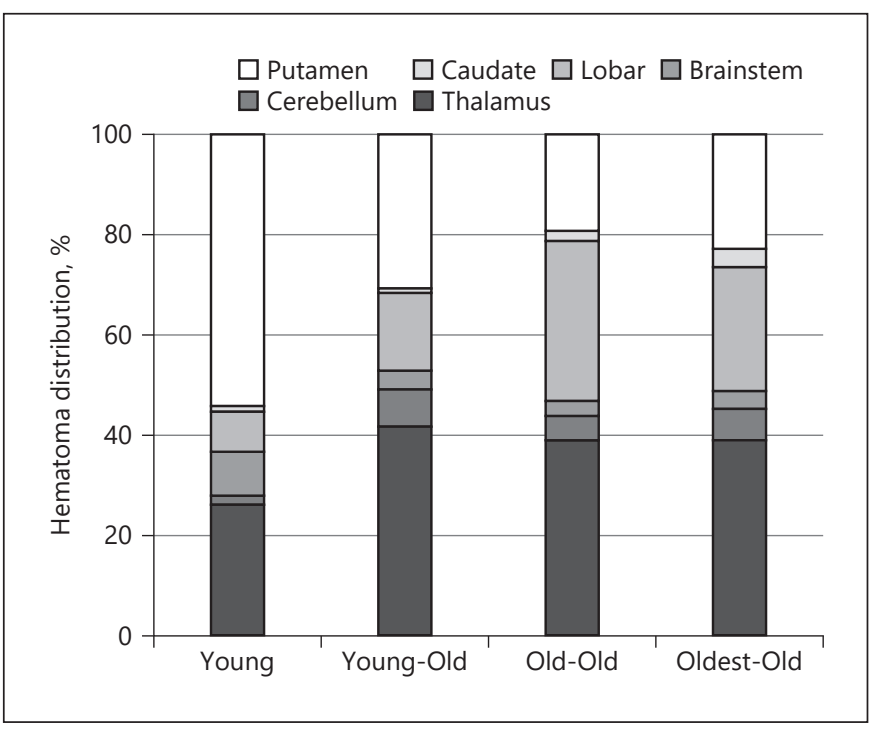

Fig. 1. Relative hematoma location by patients' age at ICH onset. $\mathrm{ICH}$, intracerebral hemorrhage.

ic hemorrhage (OR: 1.48, 95\% CI: 1.19-1.84, $p<0.001$, Table $2 \mathrm{~b}$ ) and lobar hemorrhage (OR: 1.58, 95\% CI: 1.19$2.09, p=0.002$, Table $2 \mathrm{c}$ ). The rate of putaminal hemorrhage seemed to decrease steeply with age, whereas the proportions of thalamic and lobar hemorrhages seemed to increase in young-old and old-old or older groups, respectively (Fig. 1). No significant relationships were found between advanced age and caudate, brainstem, and cerebellar hemorrhages.

Next, to assess the effect of SVD burden on hematoma location, clinical characteristics were compared among patients with different hematoma locations (online suppl. Table 1, 2). Proportion of female sex $(p=0.002)$, having hypertension ( $p=0.024)$, current drinker $(p=0.001)$, patients who received premorbid antithrombotic treatment $(p=0.036)$, values of age $(p<0.001)$, time from ICH onset to admission $(p<0.001)$, systolic $(p=0.018)$ and diastolic $(p=0.015)$ BPs, NIHSS score $(p<0.001)$, hematoma volume $(p<0.001)$, and initial hemoglobin $(p=0.006)$ were different among various hematoma locations. MRI was performed in 344 of 444 (77\%) patients during admission, a median of 1 day (IQR 1-5 days) from onset. The inter-rater reproducibility for each SVD feature showed moderate to good (Cohen's kappa statistics: presence of microbleed[s], 0.65; presence of lacune[s], 0.62; prominent perivascular space, 0.50 ; and severe $\mathrm{WMH}$, 0.64) agreement. Each SVD marker tended to differ among various hematoma locations, especially $\mathrm{WMH}$ and microbleed (online suppl. Table 2). The total SVD burden score was also different among various hematoma locations $(p<0.001)$, and multivariate logistic regression analysis showed that the total SVD burden score was independently associated with thalamic hemorrhage (OR: 1.27, 95\% CI: $1.01-1.59, p=0.045$ for 1-point increment, Table 3), even after adjusting by age. The total SVD burden score was independently and negatively related to lobar hemorrhage (OR: 0.74, 95\% CI: 0.55-0.99, $p=0.042$ ) and showed a nonsignificant tendency for a negative association with putaminal hemorrhage (OR: $0.91,95 \% \mathrm{CI}$ : $0.73-1.14, p=0.395$, Table 3 ) on multivariate analyses. Indeed, the proportion of thalamic hemorrhage rose and the rate of lobar hemorrhage decreased as the total SVD burden score increased in the total cohort and most sub- 

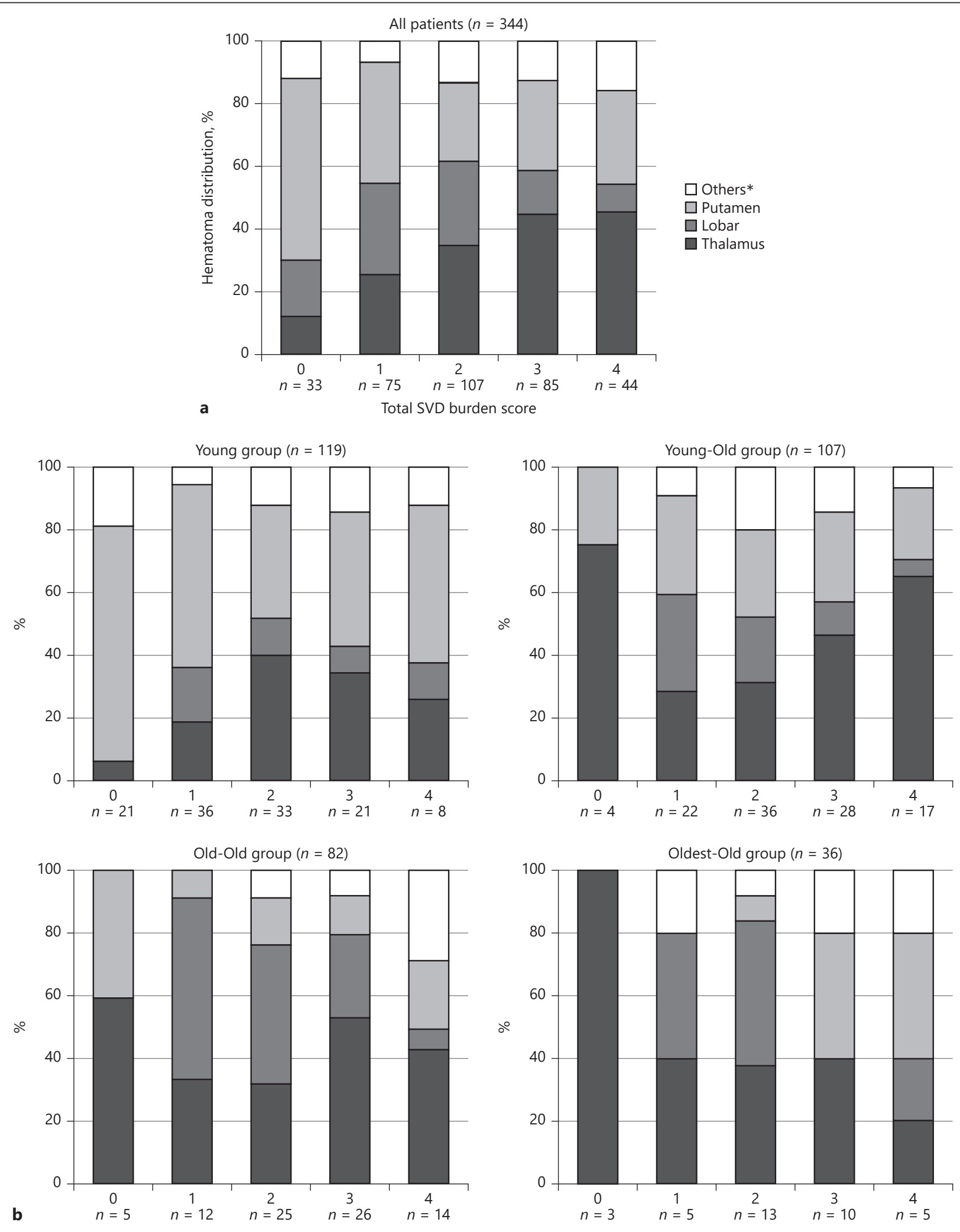

Fig. 2. Distribution of hematoma locations according to the total SVD burden score in all patients (a) and age-stratified subgroups (b). SVD, small-vessel disease. * Others include caudate, brainstem, and cerebellar hemorrhages. 
groups (Fig. 2; online suppl. Fig. 2), although some subgroups had only a few patients. The independent associations between advanced age and putaminal (OR: 0.52, $p<$ 0.001 ), thalamic (OR: $1.60, p<0.001$ ), and lobar (OR: $1.77, p \leq 0.001$ ) hemorrhages and the independent relationship between the total SVD burden score and thalamic (OR: $1.42, p=0.006$ ) hemorrhage were not changed when analyzing only patients with first-ever stroke (those without a past history of stroke, $n=366$ ). SVD score without microbleeds component was not associated with hematoma locations, but modified SVD score was independently related to thalamic (OR: 1.29, $p=0.029)$ and lobar (OR: $0.73, p=0.029$ ) hemorrhages, when using modified SVD score which removed the effect of lobar microbleeds (online suppl. Table 3).

\section{Discussion}

The present study showed that putaminal hemorrhage was independently and negatively related to advanced age, whereas advanced age was an independent factor associated with thalamic and lobar hemorrhages in consecutive patients with acute, spontaneous ICH. Cerebral SVD burden had a positive correlation with thalamic hemorrhage but a negative association with lobar hemorrhage. Advanced age was an independent factor associated with putaminal, thalamic, and lobar hemorrhages. These associations between aging and relative increase or decrease in specific brain locations of hemorrhage have been little known previously, except for lobar hemorrhage, probably because few old patients were included in the previous study [4]. A few past studies reported that patients with thalamic hemorrhage were older than those with putaminal hemorrhage, but almost all of the results were from univariate analyses $[6,7,10,12]$. One more possible explanation is that most of the past studies treated thalamic and putaminal hemorrhages together as "deep" ICH [25]. Putaminal hemorrhage occurs in young patients (mainly $<65$ years), whereas thalamic or lobar hemorrhage develops in old patients (from the results of the present study, older than 65 years for thalamic hemorrhage and older than 75 years for lobar hemorrhage, Fig. 1), and considering thalamic and putaminal hemorrhages together as deep ICH should be done cautiously, especially when evaluating factors related to aging.

The prevalence and severity of SVD are also known to increase with age [17], so we hypothesized that SVD burden was associated with thalamic or lobar hemorrhage.
Interestingly, the total SVD burden score was independently associated with thalamic hemorrhage even after adjusting for age, and, surprisingly, significantly and negatively related to lobar hemorrhage. The total SVD score tended to be negatively associated with putaminal hemorrhage. Indeed, the proportion of thalamic hemorrhage grew and that of putaminal or lobar hemorrhage decreased as the total SVD burden score increased in most age-stratified subgroups (Fig. 2 and online suppl. Fig. 2). SVD burden can be used as an imaging predictor for thalamic and lobar hemorrhages.

The occurrence of hypertensive ICH is considered to be biphasic, with patients presenting both at the onset of hypertension and later, after developing considerable wear and tear on penetrating brain arteries [14]. Later in the course of hypertension, degenerative changes including fibrinoid necrosis, lipohyalinosis, or microaneurysms develop, leading to the typical imaging features of SVD [16]. SVD burden can serve as imaging evidence for the duration of hypertension $[15,16]$, and the results of the present study may indicate that putaminal hemorrhage is prone to occur during the initial course of hypertension (young and low SVD burden), whereas, after a considerable hypertensive period during which enough SVD pathology can accumulate (old and high SVD burden), hypertensive hemorrhage is frequently observed in the thalamus. On the contrary, patients with CAA, which often causes lobar hemorrhage, have less history of hypertension and show lower BPs at ICH onset than those with hypertensive ICH [26]. Lobar ICH attributed to CAA is considered to develop after adequate amyloid deposition in the vessel wall [27], usually in the nonhypertensive state (old but low SVD burden). Age and SVD burden may predict the locations of hematomas in spontaneous ICH patients. Although the precise mechanisms for the differences in susceptibility to bleed according to age or SVD among various brain locations are unclear, hemodynamic differences originating from vascular anatomy [28] or differences in sensitivity to chronic hypertension or aging [29] may account for the heterogeneity.

The strengths of the present study included the relatively large number of spontaneous ICH patients, recruiting many oldest-old patients, and MRI acquisition in the acute phase of ICH. However, there are some limitations to be addressed. First, because of the cross-sectional nature of the study design, the results of the present study showed associations, not causal relationships. Moreover, this study showed only relative increases or decreases of specific hematoma locations by patients' age or SVD bur- 
den in the ICH cohort, not absolute increases or decreases. Second, due to the retrospective study design, whether and when to perform head MRI was left to the attending physicians' discretion. Consecutive patients with acute, spontaneous ICH were included in the present study, but MRI information (including total SVD burden score) was available in only $77 \%$ (344 of 444 patients) of the patients. Third, although the total cohort was relatively large, some subgroups were very small (Fig. 2). Fourth, almost all of the patients included in the present study were Asian, so the results may not be generalizable to all ICH patients; indeed, most of the past studies that showed that patients with thalamic hemorrhage were older than those with putaminal hemorrhage were from Asian countries [7, 10, $12]$. The results of the present study generate hypotheses, rather than showing causal relationships. The present results should be confirmed by a large-scale prospective study including patients of varied ethnic backgrounds to investigate the causal relationships between hematoma location and advanced age or SVD burden.

In conclusion, putaminal, thalamic, and lobar hemorrhages tend to occur at specific ages and in specific SVD states: putaminal in young and low SVD burden; thalamic in old and high SVD burden; and lobar in old and low SVD burden. The combination of age and SVD burden was associated with hematoma location. Susceptibility to bleeds in the early or longstanding phase of hypertension seems to be considerably different among brain locations, even between the putamen and thalamus, which are both supplied by perforating arteries and often regarded together as deep ICH when hemorrhage occurs. Unraveling the underlying mechanisms of this heterogeneity is urgently needed for better understanding and developing future prevention strategies for $\mathrm{ICH}$.

\section{Acknowledgements}

The authors would like to express their deepest gratitude to all members of the SU and radiology and emergency departments. They would also like to thank Asami Takeuchi for her assistance with database registration.

\section{Statement of Ethics}

This study was approved by the institutional Ethics Committee (No. 26-10-398). Written, informed consent was obtained from all patients or their next of kin.

\section{Conflict of Interest Statement}

The authors have no conflicts of interest to disclose.

\section{Funding Sources}

This work was partly supported by JSPS KAKENHI (Grant No. JP19K23985).

\section{Author Contributions}

Yuki Sakamoto: designed and conceptualized the study, analyzed the data, conducted statistical analyses, and drafted the manuscript. Takahiro Sato: analyzed the data. Chikako Nito: interpreted the data. Yasuhiro Nishiyama: interpreted the data. Satoshi Suda: played a major role in the acquisition of the data. Noriko Matsumoto: played a major role in the acquisition of the data. Junya Aoki: played a major role in the acquisition of the data. Tomonari Saito: played a major role in the acquisition of the data. Kentaro Suzuki: played a major role in the acquisition of the data. Takehiro Katano: played a major role in the acquisition of the data. Kazumi Kimura: played a major role in the acquisition of the data.

\section{References}

1 Sacco S, Marini C, Toni D, Olivieri L, Carolei A. Incidence and 10-year survival of intracerebral hemorrhage in a population-based registry. Stroke. 2009;40(2):394-9.

2 Krishnamurthi RV, Feigin VL, Forouzanfar $\mathrm{MH}$, Mensah GA, Connor M, Bennett DA, et al. Global and regional burden of first-ever ischaemic and haemorrhagic stroke during 1990-2010: findings from the Global Burden of Disease Study 2010. Lancet Global Health. 2013;1:e259-81.

3 Stein M, Misselwitz B, Hamann GF, Scharbrodt W, Schummer DI, Oertel MF. Intracerebral hemorrhage in the very old: future demographic trends of an aging population. Stroke. 2012;43(4):1126-8.
4 Arboix A, Vall-Llosera A, García-Eroles L, Massons J, Oliveres M, Targa C. Clinical features and functional outcome of intracerebral hemorrhage in patients aged 85 and older. J Am Geriatr Soc. 2002;50(3):449-54.

5 Inoue Y, Miyashita F, Minematsu K, Toyoda K. Clinical characteristics and outcomes of intracerebral hemorrhage in very elderly. J Stroke Cerebrovasc Dis. 2018;27(1):97-102.

6 Chiquete E, Ruiz-Sandoval MC, AlvarezPalazuelos LE, Padilla-Martínez JJ, GonzálezCornejo S, Ruiz-Sandoval JL. Hypertensive intracerebral hemorrhage in the very elderly. Cerebrovasc Dis. 2007;24(2-3):196-201.
7 Itabashi R, Yasaka M, Kuwashiro T, Nakagaki $\mathrm{H}$, Miyashita F, Naritomi H, et al. Location of acute brain hemorrhage in patients undergoing antithrombotic therapy. J Neurol Sci. 2009;280(1-2):87-9.

8 Steiner T, Petersson J, Al-Shahi Salman R, Christensen H, Cordonnier C, Csiba L, et al. European research priorities for intracerebral haemorrhage. Cerebrovasc Dis. 2011;32(5): 409-19.

9 Forti P, Maioli F, Domenico Spampinato M, Barbara C, Nativio V, Coveri M, et al. The effect of age on characteristics and mortality of intracerebral hemorrhage in the oldest-old. Cerebrovasc Dis. 2016;42(5-6):485-92. 
10 Gotoh S, Hata J, Ninomiya T, Hirakawa Y, Nagata M, Mukai N, et al. Trends in the incidence and survival of intracerebral hemorrhage by its location in a Japanese community. Circ J. 2014;78(2):403-9.

11 Hirohata M, Yoshita M, Ishida C, Ikeda SI, Tamaoka A, Kuzuhara S, et al. Clinical features of non-hypertensive lobar intracerebral hemorrhage related to cerebral amyloid angiopathy. Eur J Neurol. 2010;17(6):823-9.

12 Suzuki K, Kutsuzawa T, Takita K, Ito M, Sakamoto T, Hirayama A, et al. Clinico-epidemiologic study of stroke in Akita, Japan. Stroke. 1987;18(2):402-6.

13 Delcourt C, Sato S, Zhang S, Sandset EC, Zheng D, Chen X, et al. Intracerebral hemorrhage location and outcome among INTERACT2 participants. Neurology. 2017;88(15): 1408-14.

14 Caplan L. Intracerebral hemorrhage revisited. Neurology. 1988;38(4):624-7.

15 Furuta A, Ishii N, Nishihara Y, Horie A. Medullary arteries in aging and dementia. Stroke. 1991;22(4):442-6.

16 Pantoni L. Cerebral small vessel disease: from pathogenesis and clinical characteristics to therapeutic challenges. Lancet Neurol. 2010; 9(7):689-701.

17 Hilal S, Mok V, Youn YC, Wong A, Ikram MK, Chen CL. Prevalence, risk factors and consequences of cerebral small vessel diseas- es: data from three Asian countries. J Neurol Neurosurg Psychiatry. 2017;88(8):669-74.

18 Sakamoto Y, Nito C, Nishiyama Y, Suda S, Matsumoto N, Aoki J, et al. Safety of anticoagulant therapy including direct oral anticoagulants in patients with acute spontaneous intracerebral hemorrhage. Circ J. 2019;83(2): 441-6.

19 Wardlaw JM, Smith EE, Biessels GJ, Cordonnier C, Fazekas F, Frayne R, et al. Neuroimaging standards for research into small vessel disease and its contribution to ageing and neurodegeneration. Lancet Neurol. 2013; 12(8):822-38.

20 Fazekas F, Chawluk JB, Alavi A, Hurtig HI, Zimmerman RA. MR signal abnormalities at $1.5 \mathrm{~T}$ in Alzheimer's dementia and normal aging. AJR Am J Roentgenol. 1987;149(2):3516.

21 Doubal FN, MacLullich AM, Ferguson KJ, Dennis MS, Wardlaw JM. Enlarged perivascular spaces on MRI are a feature of cerebral small vessel disease. Stroke. 2010;41(3):4504.

22 Staals J, Makin SD, Doubal FN, Dennis MS, Wardlaw JM. Stroke subtype, vascular risk factors, and total MRI brain small-vessel disease burden. Neurology. 2014;83(14):122834.

23 Jung YH, Jang H, Park SB, Choe YS, Park Y, Kang SH, et al. Strictly lobar microbleeds re- flect amyloid angiopathy regardless of cerebral and cerebellar compartments. Stroke. 2020;51(12):3600-7.

24 Graff-Radford J, Botha H, Rabinstein AA, Gunter JL, Przybelski SA, Lesnick T, et al. Cerebral microbleeds: prevalence and relationship to amyloid burden. Neurology. 2019; 92(3):e253-62.

25 Sreekrishnan A, Dearborn JL, Greer DM, Shi FD, Hwang DY, Leasure AC, et al. Intracerebral hemorrhage location and functional outcomes of patients: a systematic literature review and meta-analysis. Neurocrit Care. 2016; 25(3):384-91.

26 Itoh Y, Yamada M, Hayakawa M, Otomo E, Miyatake T. Cerebral amyloid angiopathy: a significant cause of cerebellar as well as lobar cerebral hemorrhage in the elderly. J Neurol Sci. 1993;116(2):135-41.

27 Mandybur TI. Cerebral amyloid angiopathy: the vascular pathology and complications. J Neuropathol Exp Neurol. 1986;45(1):79-90.

28 Blanco PJ, Müller LO, Spence JD. Blood pressure gradients in cerebral arteries: a clue to pathogenesis of cerebral small vessel disease. Stroke Vasc Neurol. 2017;2(3):108-17.

29 Yamaguchi T, Miyashita T, Minematsu K, Yamaguchi S, Moriyasu H. Incidence of thalamic infarction and hemorrhage, and their distribution in the thalamus (in Japanese with English abstract). Jpn J Stroke. 1987;9:513-8. 